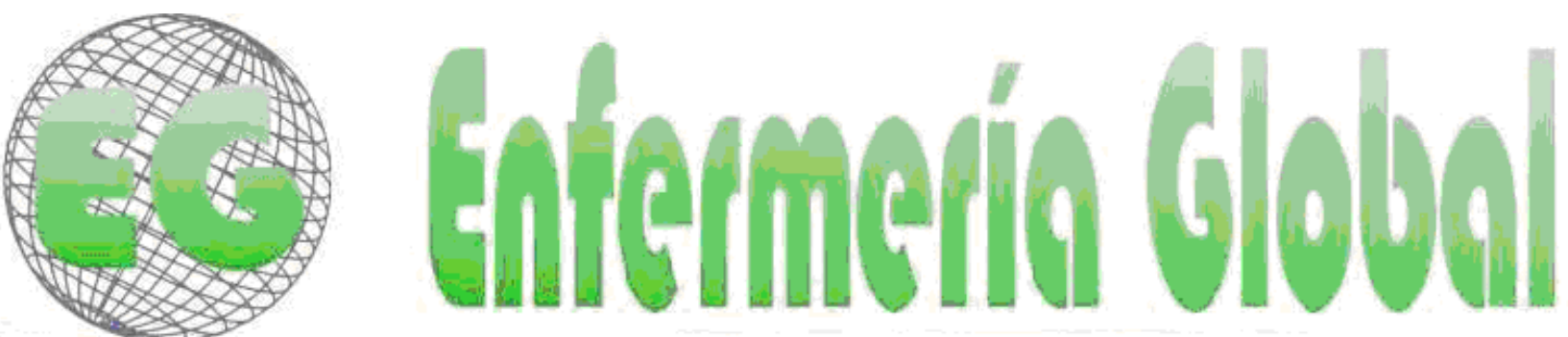

$\mathrm{N}^{\circ} 16$

Revista electrónica cuatrimestral de Enfermeria

Junio 2009

www.um.es/egloball

CLÍNIGA

\title{
CONCEPCIONES, CONOCIMIENTOS Y PRÁCTICAS DE LOS ENFERMEROS AL CUIDADO DE LOS SUJETOS CON DIAGNÓSTICO DE DEPRESIÓN: UNA MIRADA AL TERRITORIO DE LA ATENCIÓN PRIMARIA EN SALUD
}

CONCEPCÕES, CONHECIMENTOS E PRÁTICAS DOS ENFERMEIROS AO CUIDAR DE SUJEITOS COM DIAGNÓSTICO DE DEPRESSÃO: UM OLHAR PARA O TERRITÓRIO DA ATENÇÃO PRIMÁRIA À SAÚDE

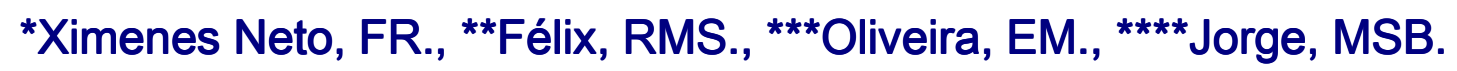

*Profesor del Curso de Graduación en Enfermería de la Universidad Estatal del Valle del Acaraú-UVA. Preceptor de la Escuela de Formación en Salud de la Familia Visconde de Sabóia, Sobral-Ceará. Grupo de Estudios y Pesquisas en Administración en Salud y Gerencia de Enfermería-GEPAG da Universidad Federal de Sao Paulo-UNIFESP. " Enfermera Graduada por la UVA. ${ }^{* * *}$ Doctora en Enfermería. Profesora del Curso de Graduación en Enfermería de la Universidad Estatal del Valle del Acaraú-UVA. ${ }^{* * * * * *}$ Doctora en Enfermería. Profesora Titular en Enfermería en Salud Mental. Coordinadora del Curso de Maestrado Académico en Salud Pública de la Universidad Estatal del Ceará-UECE. Brasil.

Palabras clave: Salud Mental; Depresión; Enfermero; Estrategia Salud de la Familia; Cuidado

Palavras-chave: Saúde Mental; Depressão; Enfermeiro; Estratégia Saúde da Família; Cuidado

\section{RESUMEN}

El estudio tiene como objetivo: caracterizar el perfil socio-demográfico y educacional de los enfermeros de la ESF; verificar el conocimiento de los enfermeros sobre la depresión; identificar las dificultades enfrentadas por los enfermeros en la efectividad de la asistencia y la promoción de la salud de los sujetos portadores de depresión; y identificar las necesidades de calificación para actuar de forma competente en el cuidado al portador de depresión. Se trata de un estudio exploratorio - descriptivo y analítico con integración de abordajes cualitativa y cuantitativa, realizado con 30 enfermeros de la Estrategia Salud de la Familia de la sede de la provincia de Sobral - CE. De los enfermeros de la muestra $93 \%$ son mujeres, con la edad entre veinte dos a treinta años $60 \%$, con un a diez años de graduados $67 \%$, y poseyendo una Residencia en la modalidad de Especialización en Salud de la Familia. Los resultados evidencian que los enfermeros relataron tener como mayores dificultades para la efectividad de la asistencia y promoción de la salud de los portadores de depresión, la inexistencia de calificación. Vale resaltar que considerar encerrada o concluida una discusión 
como la que tratamos de construir en este trabajo podría desfigurar el carácter dinámico, la formación de las ideas y resultados aquí presentados.

\section{RESUMO}

O estudo objetiva: caracterizar o perfil sócio-demográfico e educacional dos enfermeiros da ESF; verificar o conhecimento dos enfermeiros sobre depressão; identificar as dificuldades enfrentadas pelos enfermeiros na efetivação da assistência e promoção à saúde dos sujeitos portadores de depressão; e identificar as necessidades de qualificação para atuar de forma competente no cuidado ao portador de depressão. Trata-se de um estudo exploratório-descritivo e analítico com integração de abordagens qualitativa e quantitativa, realizado com 30 enfermeiros da Estratégia Saúde da Família da sede do município de Sobral - CE. Dos enfermeiros da amostra 93\% são mulheres, com idade entre vinte dois a trinta anos $60 \%$, com um a dez anos de graduados $67 \%$, e possuindo Residência na modalidade de Especialização em Saúde da Família. Os resultados evidenciam que os enfermeiros relataram ter como maiores dificuldades para efetivação da assistência e promoção à saúde dos portadores de depressão, a inexistência de qualificação. Vale ressaltar que considerar encerrada ou concluída uma discussão como a que tentamos construir neste trabalho poderia desfigurar o caráter dinâmico e em formação das idéias e resultados aqui apresentados

\section{ABSTRACT}

The objective of the study is To characterize the socio-demographic and education of nurses in the HSF; to verify the nurses' knowledge of depression; to identify the difficulties faced by nurses in the effectiveness of attendance and promotion of health of individuals with depression; and to prioritize the need for treatment in order to act in a competent way for the care of the individual with depression. It is an exploratory-descriptive and analytic study with a qualitative and quantitative integration of approaches, carried out with 30 nurses from the Health Strategy of the Family in the headquarters of the municipal district of Sobral - CE. Of the sample nurses $93 \%$ were women, $60 \%$ aged 22 to 30 years, $67 \%$ with 1 to 10 years experience since graduation, and having a residence expertise Family Health. The results showed that the nurses reported the greatest difficulty as the inexistence of qualification for the effectiveness of assistance and promotion of health of the individuals with depression. It is worth pointing out that to consider finished the discussion that we tried to create in this work, could deform the dynamic character, the formation of ideas, and results herein presented.

\section{INTRODUCCIÓN}

La depresión es, reconocidamente, un problema de Salud Pública. Es uno de los procesos patológicos de mayor frecuencia en la Atención Primaria de la Salud, con cerca de 10\% de todas las nuevas consultas. Afecta a la población en general, siendo de alta inhabilitación e interfiere de modo decisivo e intenso en la vida personal, profesional, social y económica de los portadores. Es potencialmente letal, pues en casos graves, existe el riesgo continuo de suicidio. $^{1}$

Muchos sujetos sufren en silencio, ya sea porque no se consultan, ya sea porque los profesionales no diagnostican, ni los tratan adecuadamente. Es un factor de riesgo para otras enfermedades, pues las formas moderadas de depresión pueden presentarse marcadas por otras dolencias, tales como dolor de cabeza persistente, dispepsia, falta de apetito, estreñimiento, mal gusto en la boca, generando grandes costos al sistema de salud y a la sociedad. $^{2}$

Durante toda la vida, un 31 a $50 \%$ de la población brasileña presenta, al menos, un episodio de trastorno mental y cerca de 20 a $40 \%$ necesitan, en virtud de esos trastornos, de algún 
tipo de ayuda profesional. Los sujetos deprimidos pueden no buscar un servicio de salud debido a los propios síntomas causadores de la enfermedad, como falta de fuerza, indecisión, inseguridad y culpabilidad. Además del estigma asociado a la enfermedad mental, se verifica que hay falta de atención de los profesionales de salud a los problemas emocionales ${ }^{3}$. Hoy son cuatrocientos millones de sujetos los que sufren, en el mundo, de problemas psicológicos. ${ }^{4}$

Cada día aumenta el número de portadores de depresión, siendo necesario crear medios para atender a esa clientela en todos los niveles de atención de la salud, destacando la APS, que compone la red de asistencia y dispone un contacto inicial con atención, si no cualificado, sí capaz de encaminarlo al servicio especializado.

En la Estrategia Salud de la Familia-ESF, el enfermero desempeña un importante papel en el abordaje y en la atención de los sujetos con enfermedades mentales, mayormente aquellos con depresión, dado que el enfermero en todos los tipos de institución de salud tiene la responsabilidad de reconocer e intervenir apropiadamente en los casos en que el individuo está sufriendo de un trastorno de humor. ${ }^{5}$

Según autores, la ESF a la atención a la salud mental desarrollada por el enfermero debe incluir la detección de la demanda, diagnóstico simple, manejo de los casos auto-limitantes como la depresión leve, la colecta de datos esenciales, direccionamiento de los casos que no pueden ser acompañados en este nivel, trabajo con familias y comunidad, además del apoyo y búsqueda de socialización. ${ }^{6}$

Se debe considerar que la relación entre los servicios de la APS y el de referencia en salud mental comunitaria debe estar definida entre las políticas locales de salud y que los límites de intervención de un nivel a otro pueden ser distendidos. Los niveles de atención están interrelacionados, articulados e intercambiables. ${ }^{7}$

La nueva orientación del modelo asistencial está imponiendo la implantación gradual y creciente de una red de cuidados cuya organización exige una compleja estructura de servicio comunitario articulado en recursos territoriales, culturales, sociales; enfermería de salud mental en hospital general; residencia terapéutica y cooperativa social.

En este contexto, se encuentra la tarea radical de construcción de una nueva acción de salud, dando complejidad al tipo de respuesta y al tipo de oferta de producto de salud mental, en que los trabajadores de salud tienen el desafío de disponer el saber técnico, adquirido en la formación profesional, y una habilidad dada en el trato de la diversidad e imprevisión. Se trata de la ruptura paradigmática, conceptual y ética. ${ }^{8}$

En este escenario, el enfermero ocupa una posición singular en los servicios de salud, pues, generalmente, él es el primer profesional que tiene contacto con la persona que busca atención de estos servicios. Las razones para que los trabajadores de Enfermería se ocupen de los trastornos afectivos y sus potenciales riesgos están relacionados con su experiencia y su preparación profesional. Además, los sujetos se sienten menos intimidados por los enfermeros que por los otros trabajadores de salud y los aceptan más fácilmente, su asistencia no se limita a ayudar al sujeto, sino también orientar la familia y la comunidad. ${ }^{1}$

Al entender que el cuidado al portador de trastornos mentales está siempre presente en la vida profesional del enfermero, independientemente de su área de actuación y por considerar que los servicios básicos de salud son locales de detección precoz y acción preventiva de los trastornos, decidimos trabajar con esa temática. También, por existir pocos trabajos 
relacionados a este asunto en la APS y con vistas a formular propuestas para mejora del trabajo dentro de esta problemática.

Así, el estudio tiene como objetivos: caracterizar el perfil socio-demográfico y educacional de los enfermeros de la ESF; verificar el conocimiento de los enfermeros sobre depresión; identificar las dificultades afrontadas por los enfermeros al efectuar la asistencia y promoción de la salud de los sujetos portadores de depresión; e identificar las necesidades de cualificación para actuar de forma competente en el cuidado al portador de depresión.

\section{METODOLOGÍA}

Se trata de un estudio exploratorio-descriptivo y analítico con integración de abordajes cualitativo y cuantitativo. La población se constituye de 92 enfermeros de la ESF de SobralCE. En cuanto a la muestra estaba formada por 30 enfermeros de ESF de la sede de la Provincia de Sobral. Inicialmente se pretendió realizar la pesquisa con todos los enfermeros de la Sede de la Provincia de Sobral; muchos alegaron no poder responder el cuestionario por falta de tiempo y esperar para que todos respondiesen cuando tuviesen tiempo, atrasaría mucho nuestro estudio, lo que nos decidió a hacer la muestra con 30 profesionales.

La pesquisa fue desarrollada en el periodo de mayo a julio de 2006. Para la colecta de datos fue utilizado un instrumento del tipo cuestionario, con preguntas abiertas y cerradas, teniendo los siguientes contenidos: datos de identificación, socio-demográficos y relativos a la atención prestada por los enfermeros a los sujetos portadores de depresión. El cuestionario fue entregado a los profesionales que consintieron participar en la pesquisa, después de firmado el Término de Consentimiento Pos-Informado. Estos tuvieron un plazo de una semana para la devolución del mismo.

Los datos fueron analizados en forma de categorías, además del agrupamiento en tablas y discusión de puntos relevantes, siempre haciendo uso de literatura especializada.

Resaltamos que durante todas las fases del estudio fueron respetados los aspectos éticos de la pesquisa involucrando seres humanos conforme Resolución № 196/1996 del Consejo Nacional de Salud.

\section{RESULTADOS DE LA PESQUISA}

\subsection{Características socio-demográficas educacionales}

Tabla 1 Perfil socio-demográfico y educacional de los enfermeros de la Estrategia Salud de la Familia. Sobral - Ceará - Brasil, jul. 2006.

\begin{tabular}{lrl}
\hline Variables & $N^{\circ}$ & $\%$ \\
\hline Sexo & 28 & 93,0 \\
Femenino & 2 & 7,0 \\
Masculino & & \\
\hline Edad & 18 & 60,0 \\
22 a 30 & 5 & 17,0 \\
31 a 40 & 4 & 13,0 \\
41 a 50 & 3 & 10,0 \\
\hline
\end{tabular}




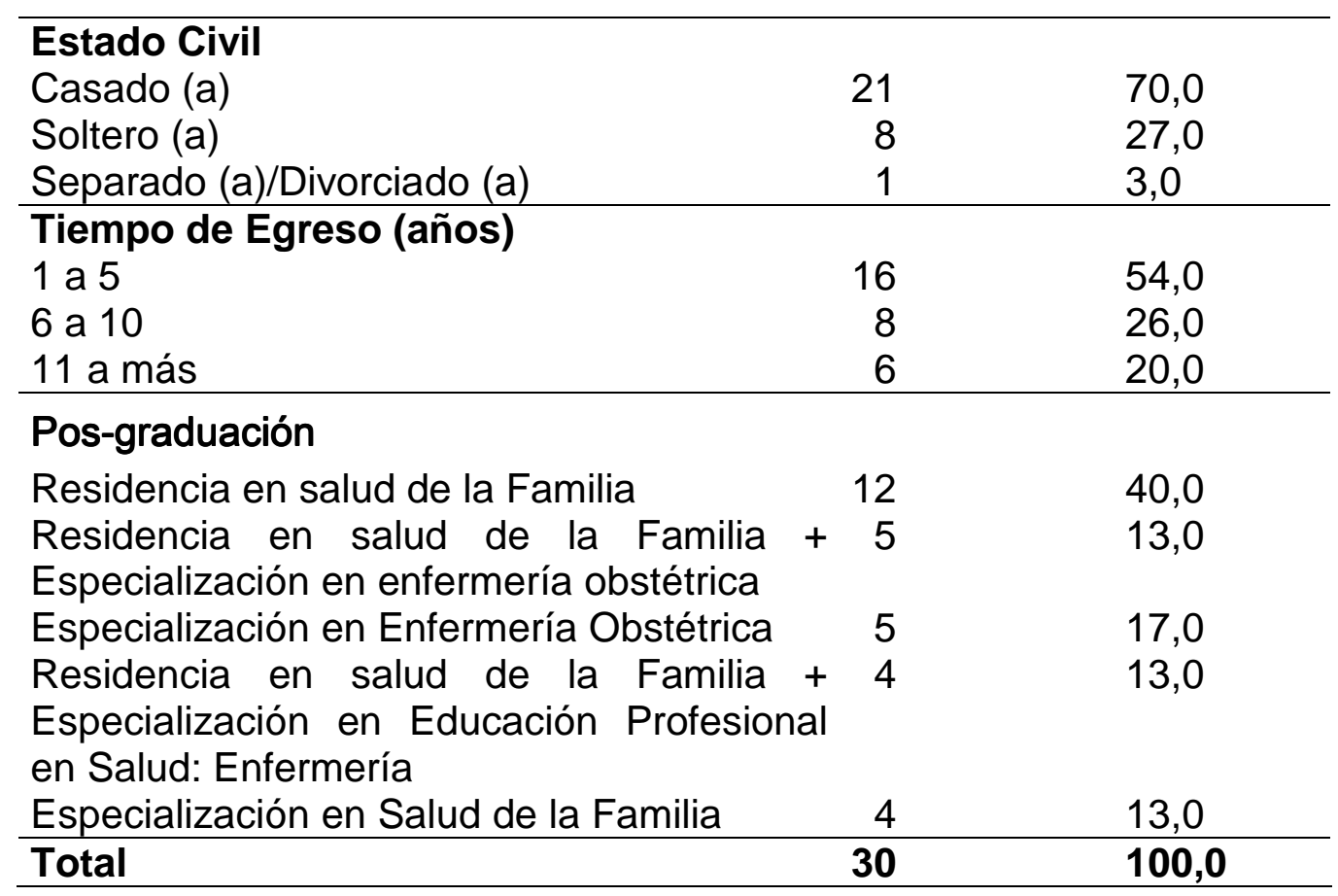

De los enfermeros investigados 28 (93\%) son del sexo femenino. Las mujeres a lo largo del tiempo son mayoría en la inserción en Cursos de Graduación en Enfermería, aunque la creciente participación de interesados es del sexo masculino.

Admitiendo como marco de nacimiento de la enfermería moderna la fecha de 9 de Julio de 1860, cuando fueron aceptadas las matrículas de 15 candidatas en la Escuela Nightingale, se puede identificar la reproducción de la división del trabajo y la presencia única de mujeres, que eran denominadas lady-nurses y nurses ${ }^{9}$.

Estas mujeres repasaban sus deberes a sus hijas y estas a la suyas y así sucesivamente, de generación en generación, de ahí el dominio del sexo femenino.

Según el Ministerio de Educación ${ }^{10}$, las mujeres son mayoría en las universidades brasileras y en los cursos del área de salud, este hecho se hace más evidente aún en los cursos de graduación en fono audición, servicio social, terapia ocupacional y nutrición, donde representan más de $90 \%$ de los estudiantes.

Sólo en el curso de educación física el número de hombres es superior, con 54,7\% de los concluyentes en 2004. En el caso de la Graduación en medicina, las mujeres son mayoría en el ingreso, pero el número de concluyentes hombres es superior $52 \%$ frente a $48 \%$ respectivamente.

Se constató que la Provincia de Sobral absorbe gran parte de los enfermeros recién egresados, pues en su cuadro de profesionales prevalecen los jóvenes y con poco tiempo de graduación.

Analizando los datos referentes a la edad de los enfermeros que actúan en la ESF de la sede de la Provincia de Sobral, podemos observar un predominio de profesionales jóvenes en el rango de edad de 22 a 30 años con 60\% (18). Este número diminuyó conforme aumenta el rango de edad. Podemos observar en cuanto al tiempo de egreso de los sujetos de la pesquisa, que una gran parte, $80 \%$ (24) se graduó de 1 a 10 años atrás. 
En lo que respecta a los sujetos de la investigación, percibimos que la mayoría ya dispone de por lo menos una Especialización o está cursando la Residencia de Salud de la Familia, a pesar de que la mayoría tiene poco tiempo de experiencia, 40\% (12) poseen o están cursando la Residencia de Salud de la Familia.

\subsection{Conceptos sobre depresión}

Aquí fueron separadas en categorías las respuestas de los enfermeros, cuando se les preguntó sobre lo que entendían por depresión, lo que se percibió es que no dieron un concepto bien resumido, describieron la enfermedad citando sólo parte de la sintomatología.

Lo que se oye hablar es que la depresión es la enfermedad del alma (Enfermero 1). Disturbio de Comportamiento (Enfermero 3).

Es algo sentido por la persona que hace que el ser humano sea incapaz de realizar sus actividades como de costumbre (Enfermero 6).

Estado general de tristeza donde la persona pierde la alegría de vivir (Enfermero 8). Se manifiesta por apatía, desanimo, llanto fácil, postración en casos crónicos (Enfermero 9).

Cuadro de baja auto-estima, cuando la persona no tiene ganas de nada, ni de comer, alimentarse (Enfermero 10).

Proceso psicológico en el cual se remite a alteraciones de intensidad comportamiento diferente, acarreando desinterés por la vida y disgusto por los hábitos, debe ser tratado con psicofármacos (Enfermero 18).

Disturbio Afectivo, que lleva a un desequilibrio psíquico, consecuencia de un desequilibrio físico, ocasionado a lo que se sabe por alteraciones en los neurotransmisores en las sinapsis, la persona debe participar de terapias de grupo (Enfermero 19).

De los enfermeros investigados, un cantidad representativa, aproximadamente $80 \%$, dieron solamente una descripción resumida cuando fueron preguntados sobre lo que entendían por depresión, lo que no deja de ser válido para una evaluación ya que se trataba de una pregunta de cuño subjetivo para ellos.

Bien diferente de la tristeza o de bajón, la depresión es el resultado de una alteración de la acción de neurotransmisores en el cerebro. Es una enfermedad con síntomas bien específicos y puede ser caracterizada cuando estos perduran como mínimo dos semanas, clasificada como un trastorno del humor. ${ }^{11}$

Al describir lo que entienden sobre depresión, algunos enfermeros citaron parte de la sintomatología como definición, dejando de hablar de otros aspectos que engloban la enfermedad. Es común que las personas definan una enfermedad por medio de síntomas que caracterizan su estado, pero se sabe que una patología envuelve más aspectos y los profesionales de salud deben ser capaces de describirlos.

El sujeto deprimido se siente incapaz, desinteresado por las cosas, con su energía vital disminuida. Los sentimientos son tantos y tan confusos que a veces se considera la depresión como si estuviese "anestesiado", sin sentimientos. Puede haber tristeza intensa, llanto fácil, irritación con pequeños problemas, sensación de menos valía, ganas de abandonar todo y todos. Las actividades antes efectuadas naturalmente, como bañarse, vestirse, cuidar de sus cosas, cumplir con sus compromisos, ahora son hechas con un esfuerzo enorme. ${ }^{12}$ 
De modo general lo que se percibió fue que los enfermeros tienen conocimientos básicos sobre la depresión, que de alguna manera sirven como parámetro para una evaluación de una persona en estado depresivo.

Los síntomas de la depresión según un autor, se distinguen del pesar y de la tristeza básicamente por el trastorno de humor. Así, una persona que esté triste deberá ser capaz de mantener algún buen humor o alguna esperanza. Esta es la primera e importante diferencia; en la depresión, la experiencia emocional es mucho más intensa y duradera. La persona deprimida se siente sin esperanza, sin vitalidad, sin posibilidad de librarse de estos sentimientos y no logra mantener saludable ningún aspecto de su vida. ${ }^{13}$

Por otro lado, algunos enfermeros investigados dieron una definición más completa, mostrando que no todos tienen conocimientos restrictos sobre la depresión. La depresión puede tener causas apenas endógenas. Es como si todo llevase a la tristeza, pero así mismo el sujeto está deprimido. Sin embargo, muchas veces, el mal es desencadenado por factores como, el estrés agudo, muerte de un ente querido y una enfermedad grave. ${ }^{14}$

El tratamiento acostumbra asociar la utilización de medicamentos y psicoterapia. Los medicamentos antidepresivos son responsables de la minimización de los síntomas y el control de la angustia. La psicoterapia es un proceso conducido por un profesional especializado y se caracteriza por atenciones regulares que auxilian la desesperanza, la falta de motivación, la autoestima diminuida y la tristeza. Se atribuye este proceso a una disminución del sufrimiento psicológico, del sentimiento del adolecer y la mejora de la cualidad de vida. ${ }^{12}$

El enfermero, cuanto más conocimientos tenga en salud mental, más conciencia tendrá de su condición personal y social, de su papel de trabajador inserto en un contexto social de ciudadano en un sistema político, más apto estará para elegir instrumentos de trabajo que procuren el rescate de la misma condición de sujeto ciudadano a los sujetos con trastornos mentales, principalmente los depresivos que son la mayoría. ${ }^{6}$

\subsection{Formación y Cualificación}

Tabla 2 Formación de los enfermeros durante la graduación académica para prestar atención a los portadores de depresión. Sobral - Ceará Brasil, jul. 2006.

\begin{tabular}{lrl}
\hline Disciplinas & № & $\%$ \\
\hline Si & $\mathbf{1 6}$ & $\mathbf{5 3 , 0}$ \\
Enfermería Psiquiátrica & 9 & 30,0 \\
Salud Mental & 6 & 20,0 \\
Enfermería Geriátrica & 1 & 3,0 \\
No & $\mathbf{1 4}$ & $\mathbf{4 7 , 0}$ \\
\hline Total & $\mathbf{3 0}$ & $\mathbf{1 0 0 , 0}$ \\
\hline
\end{tabular}

Como puede verse en la Tabla 2, 16 (53\%) de los enfermeros relataron haber recibido algún tipo de capacitación durante la formación académica para prestar asistencia a los portadores de depresión, mientras 14 (47\%) casi la mitad, afirmó no haber recibido ningún tipo de capacitación. La afirmación de esos catorce enfermeros nos hace repensar cómo están siendo hechos los contenidos programáticos de la teoría y de la práctica de las disciplinas de Enfermería Psiquiátrica y Salud Colectiva, demostradas como deficitarias en capacitación para algunos graduados. 
De acuerdo con Mello, algunos estudios consideran que existen varias tendencias teóricas influenciando la práctica psiquiátrica actualmente y que hay deficiencia en el proceso de formación de enfermeros para actuar en psiquiatría, toda vez que la mayoría de los cursos no se están adecuando a los constantes cambios en el campo de la salud mental, desencadenando indefinición sobre su papel en la asistencia lo que provoca muchas veces una "fuga" o resistencia para actuar en esta área. ${ }^{15}$

Tabla 3 Cualificación experimentada por los enfermeros después del egreso para actuar en la atención a los portadores de depresión. Sobral Ceará - Brasil, jul. 2006.

\begin{tabular}{lrc}
\hline Cualificación & № & $\%$ \\
\hline Si & $\mathbf{1 0}$ & $\mathbf{3 3 , 0}$ \\
Seminarios & 5 & 17,0 \\
Especialización & 4 & 13,0 \\
Graduación & 1 & 3,0 \\
No & $\mathbf{2 0}$ & $\mathbf{6 7 , 0}$ \\
\hline Total & $\mathbf{3 0}$ & $\mathbf{1 0 0 , 0}$ \\
\hline
\end{tabular}

Se observa en la Tabla 3, un número reducido de enfermeros 10 (33\%), o sea, apenas 1/3 que hizo algún tipo de curso, para actuar con portadores de depresión, después de concluir su formación académica, se constató que la mayoría de los sujetos de la pesquisa 20 (67\%) no realizó ningún tipo de cualificación.

Estos datos comparados con los de la tabla 2 revelaron un preocupante resultado, en el que poco menos de la mitad de los sujetos de la tabla anterior no cree que recibió capacitación durante la formación. Eso nos lleva a apuntar que la asistencia a esta clientela está gravemente comprometida, así como los demás cuadros de agravios y patologías en Salud Mental, que son tan comunes en nuestra sociedad.

En la descripción de los 10 enfermeros que tuvieron algún tipo de cualificación, cinco (17\%) afirmaron haber tenido esto a través de seminarios, cuatro (13\%) a través de Especialización y uno $(3 \%)$ durante la formación.

\subsection{Capacidad para identificar las señales y síntomas de la Depresión}

Indagados sobre su capacidad para identificar una persona con depresión, la gran mayoría $24(80 \%)$ respondió que sería capaz de hacer la identificación y $6(20 \%)$ respondieron que la harían pero con dudas. Aquí se incluyen los que afirmaron no haber recibido cualificación durante la Formación en la Tabla 2, colocándose como aptos para hacer la identificación de un portador de depresión.

Con base en el DSM-IV y CID-10, los síntomas clínicos de la depresión son humor depresivo, tristeza, perdida del interés o placer, pérdida o aumento de peso significativo, insomnio o hiperinsomnio, agitación o retardo sicomotor, fatiga, sentimiento de inutilidad, culpa excesiva, indecisión, capacidad diminuida de pensar y pensamientos de muerte recurrentes ${ }^{16}$. Para Rocha ${ }^{17}$ es necesario que los profesionales de salud sepan hacer la detección precoz de ese problema en su campo de actuación, para evitar una negligencia en esa área. 


\subsection{Acompañamiento de los sujetos con diagnóstico de depresión}

Tabla 4 Acciones desarrolladas por los enfermeros en la promoción de la salud de los portadores de depresión, Sobral-Ceará--Brasil, jul. 2006.

\begin{tabular}{|l|l|l|}
\hline Acciones Desarrolladas & № & $\%$ \\
\hline Identificación de los casos & 29 & 96,0 \\
\hline Busca Activa & 16 & 53,0 \\
\hline Diagnóstico precoz de complicaciones & 26 & 86,0 \\
\hline Visita Domiciliaria & 17 & 56,0 \\
\hline Control de condiciones de riesgo & 26 & 86,0 \\
\hline Estímulo a posibilidades de enfrentamiento & 23 & 73,0 \\
\hline Direccionamiento al servicio especializado & 27 & 90,0 \\
\hline Terapia comunitaria & 6 & 20,0 \\
\hline Preceptoría & 9 & 30,0 \\
\hline
\end{tabular}

Las principales actividades realizadas dirigidas a la atención de la salud de los portadores de depresión en la sede de la provincia de Sobral - Ceará son: identificación de los casos 29 $(96 \%)$ citaciones, direccionamiento al servicio especializado 27 (90\%), el control de las condiciones de riesgo para más 26 (86\%) y diagnóstico precoz de complicaciones para 26 (86\%) enfermeros, siendo esos índices porcentuales bien próximos.

Es de fundamental importancia la actuación del equipo de ESF en la identificación precoz de alteraciones de comportamientos y otras señales crónicas de agudización del cuadro de trastorno psiquiátrico, así como el acompañamiento, inclusive medicamentoso, de los sujetos con sufrimiento mental, y promover la discusión con la familia y la comunidad sobre la inserción de este usuario rompiendo o minimizando el estigma existente en relación a tales trastornos. ${ }^{5}$

Las acciones desarrolladas menos citadas fueron la busca activa 16 (53\%) y la visita domiciliaria con 17 (56\%) citaciones. Dos acciones que también deberían tener niveles más elevados, ya que una detecta y la otra permite el conocimiento de la estructuración de la vida del cliente.

Al lidiar con portadores de depresión a pesar de todo lo hecho, todavía va a parecer al final que faltó algo. Isaac elaboró elementos esenciales de un contrato terapéutico enfermerocliente, en el que los dos saben el nombre del otro; el cliente comprende el papel del enfermero; son definidas las responsabilidades de ambos; son aclaradas las metas de relación; son establecidos los locales y horarios de encuentro; son delineadas las condiciones para el término y donde la confidencialidad es discutida y asegurada. ${ }^{18}$

\subsection{Evolución y Continuidad de la Atención al Cliente con diagnóstico de Depresión}

Todos los enfermeros 30 (100\%), afirmaron marcar una reconsulta para reevaluación de los portadores de depresión. Entre las justificativas 10 (33\%) dicen que para tener un mejor acompañamiento, $8(27 \%)$ para saber como la persona está reaccionando al tratamiento indicado, 7 (23\%) para tener un control de medicación utilizada y $5(17 \%)$ para evaluar un posible cambio de conducta frente al caso.

Comparando esos datos con el hecho de que 16 (53\%) de los enfermeros afirmaran no existir un horario específico para la atención a los portadores de depresión, constatamos que 
aunque sin atención diaria y semanal hecha por Enfermería, las consultas son asumidas por los enfermeros, encajándolas dentro del horario de otros tipos de atención.

Acerca de la continuidad de la asistencia a los sujetos con diagnóstico de depresión que son referenciados para la realización de exámenes complementarios o atención especializada, constatamos que 27 (90\%) enfermeros de ESF de la sede de la provincia de Sobral afirmaron dar continuidad de asistencia y tres (10\%) no respondieron.

Al analizar las justificativas para que haya continuidad de la asistencia destacamos: para dar una mejor atención a esta clientela $12(40 \%)$, monitorear reincidencias $9(30 \%)$ y cuando hay contra-referencia 6 (20\%); siendo que 3 no respondieron.

La necesidad de insertar cuestiones de salud mental en la dinámica de la ESF se hace visible, diariamente, en las prácticas de salud, ya que la llegada de usuarios con sufrimiento psíquico en las unidades de salud es bastante frecuente, las quejas son más variadas y por eso el equipo debería estar preparado para ofrecer mayor resolución a los problemas de estos usuarios.

Se constató que hay cierto compromiso por parte de los enfermeros de la ESF en lo tocante a la calidad de la asistencia, pues la continuidad de la asistencia a los clientes necesita ser referenciada y es un buen parámetro para evaluar el compromiso de los profesionales con la persona, principalmente a los que sufren trastornos psíquicos, que necesitan toda una red de cuidados.

\subsection{Consulta Individual}

Tabla 5 Acciones realizadas con los portadores de depresión por los enfermeros durante la consulta individual. Sobral - Ceará - Brasil, jul. 2006.

\begin{tabular}{|l|l|l|}
\hline Consulta Individual & No & $\%$ \\
\hline Realiza y/o actualiza la historia clínica & 28 & 93,0 \\
\hline Investiga hábitos personales & 25 & 83,0 \\
\hline Identificar factores de riesgo & 28 & 93,0 \\
\hline Realiza examen físico & 8 & 26,0 \\
\hline Solicita y evalúa exámenes complementarios la patología & 16 & 53,0 \\
\hline Informa sobre las complicaciones de la correcto de & 23 & 76,0 \\
\hline $\begin{array}{l}\text { Ofrece informaciones sobre el uso } \\
\text { medicamentos }\end{array}$ & 93,0 \\
\hline Incentiva a adherir al tratamiento el prontuario & 28 & 93,0 \\
\hline Da orientación & 27 & 90,0 \\
\hline Registra informaciones en er & 27 & 90,0 \\
\hline
\end{tabular}

Para las acciones realizadas al sujeto con diagnóstico de depresión durante la consulta individual, la mayoría de los tópicos marcados tuvo porcentuales bien próximos, habiendo solo dos que se diferencian de los demás tópicos.

La realización y/o actualización de la historia clínica; la identificación de factores de riesgo; aclaraciones sobre el uso correcto de los medicamentos e incentivo a la adherencia al tratamiento, tuvieron 28 (93\%) citaciones. Las orientaciones y el registro de informaciones en el, prontuario 27 (90\%); la identificación de hábitos personales 25 (83\%) y la información sobre las complicaciones de patología 27 (90\%) citaciones. Con referencia a la realización 
del examen físico 8 (26\%) de los enfermeros afirmaron realizar y 16 (53\%) solicitaron exámenes complementarios.

Según un autor los profesionales de salud mental deberían ofrecer informaciones y soporte profesional necesarios para que la familia ejerza su función de agente socializador primario del ser humano. ${ }^{19}$

De esta forma, el equipo de la ESF debería estar actuando en el ambiente familiar, en el sentido de aliviar el sufrimiento de la familia y del propio usuario. Esta ayuda ofrecida por el equipo de la ESF con soporte del equipo de salud mental, podría darse por medio de visitas a los hogares, atención individual y familiar, así como reuniones de grupos en la propia comunidad asistida.

\subsection{Dificultades en la atención}

Tabla 6 Dificultades enfrentadas por los enfermeros en la asistencia y promoción de la salud de los portadores de depresión. Sobral - Ceará - Brasil, jul. 2006.

\begin{tabular}{|l|c|l|}
\hline Dificultades & № & $\%$ \\
\hline Falta de cualificación & 20 & 66,0 \\
\hline Gran Demanda & 9 & 30,0 \\
\hline Iniciar el primer abordaje & 6 & 20,0 \\
\hline Falta de Compromiso de la familia & 17 & 56,0 \\
\hline $\begin{array}{l}\text { Convencimiento para la participación en grupos de } \\
\text { terapia }\end{array}$ & 10 & 33,0 \\
\hline Dificultad de adhesión al tratamiento & 11 & 36,0 \\
\hline Falta de actividades ocupacionales en la comunidad & 2 & 6,0 \\
\hline Falta de tiempo & 10 & 33,0 \\
\hline Retorno a las consultas subsecuentes & 8 & 26,0 \\
\hline Falta de Estructura Física da UBS & 4 & 13,0 \\
\hline Ausencia del profesional Médico & 2 & 6,0 \\
\hline
\end{tabular}

En la tabla 6 fueron relacionadas las principales dificultades enfrentadas por los enfermeros de la ESF durante la ejecución de la atención al portador de depresión, entre estas, la más citada fue la falta de cualificación teniendo 20 (66\%) indicaciones. Para 17 (56\%) enfermeros la falta de compromiso de la familia es la principal dificultad; la falta de tiempo para 10 (33\%) de los sujetos investigados y $26 \%$ ) citaron la ausencia del médico.

El convencimiento para la participación en grupos de terapia tuvo 10 (33\%) citaciones; la dificultad de adhesión al tratamiento $11(36 \%)$ y el retorno a las consultas subsecuentes, fue citada por $8(26 \%)$ de los enfermeros.

En estos tres últimos tópicos las dificultades a ser enfrentadas son de la dependencia casi única del portador de depresión que, en algunos casos, es bastante reluctante en aceptar el apoyo de los profesionales y reconocerlo como agente de cambio de su cuadro, principalmente el depresivo, pues tiene una tendencia al aislamiento. 


\subsection{Necesidades de Cualificación}

Tabla 7 Necesidades de cualificación para actuar de forma competente en el cuidado al portador de depresión. Sobral - Ceará - Brasil, jul. 2006.

\begin{tabular}{|l|l|l|}
\hline Necesidades & No & $\%$ \\
\hline Participa de un proceso de educación permanente & 11 & 36,0 \\
\hline Perfeccionamiento p/conducir los casos & 8 & 26,0 \\
\hline Cursos en teoría e práctica & 10 & 33,0 \\
\hline Pasantía en servicio especializado & 7 & 23,0 \\
\hline Poder contar con a colaboración del CAPS de forma más efectiva & 2 & 6,0 \\
\hline $\begin{array}{l}\text { Poder contar con la preceptoria con preceptoria de forma más } \\
\text { asidua }\end{array}$ & 2 & 6,0 \\
\hline
\end{tabular}

Las necesidades citadas fueron la posibilidad de participación en un proceso de educación permanente en salud mental con 11 (36\%) de las citaciones; y $8(26 \%)$ citaron la realización de perfeccionamiento para conducir casos.

Los enfermeros evidenciaron necesidad de realizar cursos aplicando la teoría con la práctica, 10 (33\%) de los investigados; poder contar con el Centro de Atención PsicosocialCAPS de forma más efectiva y con la preceptoría de forma más asidua, $2(6 \%)$ de las citaciones.

La ciudadanía del portador de sufrimiento psíquico se da por medio de su (re)inserción al núcleo familiar, a la comunidad, y al trabajo. Para lograr este propósito de (re)inserción del portador de sufrimiento psíquico a la comunidad sería necesaria la participación efectiva de la red básica de salud, a través de la integración de las acciones entre los servicios sustitutivos - CAPS - y la ESF.

En las provincias que no disponen de CAPS el equipo de la ESF debería estar preparado para cubrir esta demanda. Este equipo debería recibir cualificación de un equipo de salud mental, de la propia provincia u otro, para mejorar la atención de esos profesionales a los portadores de trastorno psíquico - esquizofrenia; disturbio bipolar afectivo; depresión; neurosis; alcoholismo; drogadicción; fármaco-dependencia de benzodiazepídicos; tentativas de suicidio; problemas de orden social que afectan directa o indirectamente a los sujetos y sus familias como; violencia urbana; malos tratos a niños y ancianos, entre otros. ${ }^{20}$

También se hizo evidente la necesidad de cualificación de los sujetos, que aquí ya trazaron caminos que podrían ser seguidos para un proceso de reestructuración de la salud mental a través de los enfermeros dentro de las unidades básicas de salud.

\section{Consideraciones Finales}

El perfil de los enfermeros de la ESF, investigados está constituido en su mayoría por mujeres 28 (93\%), con edad entre los veintidós a treinta años $18(60 \%)$, con uno a diez años de egreso 20 (67\%), y tienen pos-graduación por medio de la Residencia en la modalidad de Especialización en Salud de la Familia.

Al ser indagados sobre qué entienden por depresión, una gran parte apenas dio un concepto de depresión de manera sucinta y revelaron 20 (77\%) no haber hecho ningún tipo 
de cualificación después del egreso para actuar en la atención a los portadores de depresión.

Los resultados evidencian que los enfermeros participantes de la investigación relataron tener como mayores dificultades para efectuar la asistencia y promoción de la salud a los portadores de depresión, la inexistencia de cualificación. Y como mayores necesidades, la participación en un proceso de educación permanente, con pasantías en servicios especializados donde se aplicaría la teoría y práctica.

Frente a este cuadro, percibimos que los profesionales de la ESF todavía tienen desafíos que superar, entre ellos la no preparación de los profesionales para luchar con la salud mental, constatando que cambiar el tratamiento dado al portador de depresión consiste en dos grandes acciones: ofrecer una red de cuidados que ayude al sujeto a vivir en la comunidad y construir una actitud nueva en la sociedad con relación al portador de depresión.

Creemos en la posibilidad de constituir en el cotidiano, en los enfrentamientos y en las contradicciones entre el proceso de reproducción y recreación, propios de la práctica de Enfermería, un proceso contra-hegemónico que, identificados con los preceptos de la Reforma Psiquiátrica, rescate a los actores involucrados, trabajadores y usuarios como sujetos sociales en un proceso de perfeccionamiento cotidiano, donde reside la potencialidad implícita de autonomía profesional de los enfermeros.

Vale resaltar que considerar encerrada o concluida una discusión como la tratamos de construir en este trabajo podría desfigurar el carácter dinámico y en formación de las ideas y resultados aquí presentados. Entendemos que la elaboración teórica construida a lo largo de este estudio nos reveló la necesidad de emprender más esfuerzos en el sentido de profundizar la reflexión de ampliar la discusión cada vez más a cerca del tema aquí tratado.

\section{REFERÊNCIAS}

1. OPAS/OMS. Programa de Salud Mental, División de Promoción de Salud. Modelo para la capacitación de la enfermera general en al identificación y manejo de los transtornos afectivos. Generalista I; 1997.

2. Montgomery S. Confrontando la depression. Guia del médico. New York: Pfizer Internacional; 1997.

3. Dalgalarrondo P. Psicopatologia e semiologia dos transtornos mentais. Porto Alegre: Artes Médicas; 2000.

4. Organização Pan-Americana de Saúde-OPAS. Relatório Mundial de Saúde. 2001. Disponível em: http://www.opas.org.br/mostrant.cfm?codigodest=75. Acessado em 18 mar. 2006.

5. Saraceno B, Asioli F, Tognoni G. Manual de saúde mental. 2 ed. São Paulo: Hucitec. 1999.

6. Oliveira AGB, Alessi NP. O trabalho de enfermagem em saúde mental: contradições e potencialidades atuais. Rev. Latino-Americana de Enfermagem, v.11, n.3, p.33-34. maio/jun. 2003.

7. Aranha e Silva AL. Comunicação e Enfermagem em Saúde Mental - reflexões teóricas. Rev. Latino - Am. Enfermagem, 2000.

8. Lancetti A. Saúde mental nas entranhas da metrópole. In: Jatene A, Lancetti A. Saúde loucura, saúde mental e saúde da família. 2ª ed. São Paulo: Hucitec; 2002. p. 11-52.

9. Melo CMM. Divisão social do trabalho e enfermagem. São Paulo: Cortez; 1986. 
10. Brasil. Ministério da Educação. Departamento de Ensino Superior: perfil dos ingressos. Disponível em: < http://dtr2004.educaçao.gov.br/dab>. Acessado em 18 de julho de 2006.

11. Fleck MPA, Lafer B, Souguey EB. Diretrizes da Associação Médica Brasileira para 0 tratamento da depressão. Revista Bras. Psiquiatr. v. 25, n.2. jun. 2003.

12. Rodrigues MJSF. O diagnóstico de depressão. Psicol. USP, v. 11, 2000.

13. Del Porto JA. Conceito e Diagnóstico. Rev. Bras. Psiquiatr. v. 21. supl. 1, p. 7, 1999.

14. Pontes CB. Segredos da Depressão: Guia Técnico - Prático para o tratamento. São Paulo: Editora Maltese, 1999.

15. Mello IM. Proposta de orientação para sistematização de assistência de enfermagem psiquiátrica. [dissertação]. São Paulo (SP): Escola de Enfermagem/USP; 1998.

16. Organizaçao Mundial da Saúde-OMS. Classificação de Transtornos Mentais e Comportamentais da CID - 10. Traduzido por Dorgival Caetano. Porto Alegre: Artmed, 1997.

17. Rocha RM. Enfermagem Psiquiátrica: que papel é esse? Rio de Janeiro, RJ: Instituto Franco Basalgia/Te Cora; 1994.

18. Issac RN. Série de Estudo em Enfermagem: Saúde Mental e Enfermagem Psiquiátrica. 2. ed. São Paulo: Guanabara Koogan, 1998.

19. Waidman MAP. Enfermeira e família compartilhando o processo de reiserção social do doente mental. In: Labete RC. (org). Caminhando para assistência integral. Ribeirão Preto: Scala, 1999. p. 389-407.

20. Cabral B. Estação comunidade. In: Janete A, Lancetti A. (org). Saúde mental e saúde da família. 2ª . ed. São Paulo: Hucitec, 2001. p. 137-154. 